\title{
Proof of reducing cancer risks is in the eating
}

Mounting statistical evidence suggests some foods may increase or decrease certain cancer risks. According to the National Cancer Institute (NCI), one third of all cancer deaths may be related to what we eat. As many as $80 \%$ of all cancers may be associated with our eating, drinking, and smoking behaviors alone.

April is Cancer Control Month. Recognizing the relationship between nutritional habits, obesity, and cancer, the American Cancer Society (ACS) has designated April 19 as the day of the "Great American Food Fight Against Cancer." On that day, the ACS is asking all of us to eat foods that may lower our risks for certain cancers. The ACS modeled this day after the successful "Great American Smokeout." Observed each November 15 for the past 13 years, the "Great American Smokeout" encourages all Americans to quit smoking for at least 24 hours.

As physicians, we need to reinforce the important role diet plays in the fight against cancer to all of our patients-adults, adolescents, and children. Because early learned behavior patterns set the foundation for those practiced later in life, the sooner we reach our patients with this information, the better.

Specifically, dietary recommendations designed to reduce cancer incidence include increasing dietary fiber intake, reducing daily fat intake, and increasing consumption of foods rich in vitamin A, vitamin $\mathrm{C}$, and betacarotene.

A high-fiber diet has been associated with a lower incidence of colon cancer. Unfortunately, the average adult American eats only about $11 \mathrm{~g}$ of fiber daily. The NCI recommends $20 \mathrm{~g}$ to $30 \mathrm{~g}$ each day. Whole grain breads and cereals, apples and potatoes with their skins, peaches, and pears provide good sources of natural fiber.

Similarly, the risk of breast, colon, and prostate cancer could be reduced through a low-fat diet. Approximately $40 \%$ of calories in the average adult's diet comes from fat. Most experts believe no more than $30 \%$ of our daily calorie intake should come from fat. This amount should be equally divided between saturated, monounsaturated, and polyunsaturated fats.

A low-fat diet doesn't mean giving up meat. Lean meats and skinless poultry can still be a part of our daily menu. However, baked, steamed, poached, roasted, or microwaved entrées are a healthful alternative to deep fat-fried foods.

As a precautionary measure against cancer of the larynx, esophagus, stomach, and lungs, our daily diet should feature foods rich in vitamin A, vitamin $\mathrm{C}$, and betacarotene. That means consuming more dark leafy green vegetables, and red, yellow, and orange fruits and vegetables. Likewise, a diet that includes cruciferous vegetables-broccoli, brussels sprouts, cauliflower, collards, and mustard greens - could further reduce cancer risks.

The risk of esophageal and stomach cancer may be reduced by simply avoiding salt and foods that are nitrite-cured and smoked. Finally, limiting alcohol consumption to one to two drinks daily may reduce the risk of oral, esophageal, and liver cancer.

Eating healthy doesn't have to be a chore, notes ACS President Robert J. Schweitzer, MD. While Dr Schweitzer admits dietary changes are not a "magic potion" to prevent cancer, such changes can help in the fight against cancer.

Time and again we are reminded of the importance of health promotion and disease prevention, the tenet on which the practice of osteopathic medicine is built. We join Dr Schweitzer and the ACS in the "Great American Food Fight Against Cancer." For more information about this and other activities planned for Cancer Control Month, contact your local ACS chapter, or call 1-800-ACS2345.

Bon appétit! Bonne Santé!

THOMAS WESLEY ALLEN, DO Editor in Chief 


\section{Straight.}

GORE-TEX ${ }^{\circledR}$ Vascular Grafts have

been "consistently reported

to have 24-month patency rates of

over $70 \%$." In addition, "the

chance of successful revision at

each failure is greater than $50 \% .{ }^{\prime 2}$

The straight graft is available

in $5 \mathrm{~mm}, 6 \mathrm{~mm}$, and $8 \mathrm{~mm}$

diameters with an optional orientation line.

1. Bennion RS and Wilson SE. Hemodialysis and Vascular Access. Chapter 27 in Moore WS, ed. Vascular Surgery, Orlando, FL: Grune \& Stratton, Inc.; 1986: 791-830.

2. Etheredge EE et al. Salvage operations for malfunctioning PTFE hemodialysis access grafts. Surg 1983; 94:464-470.

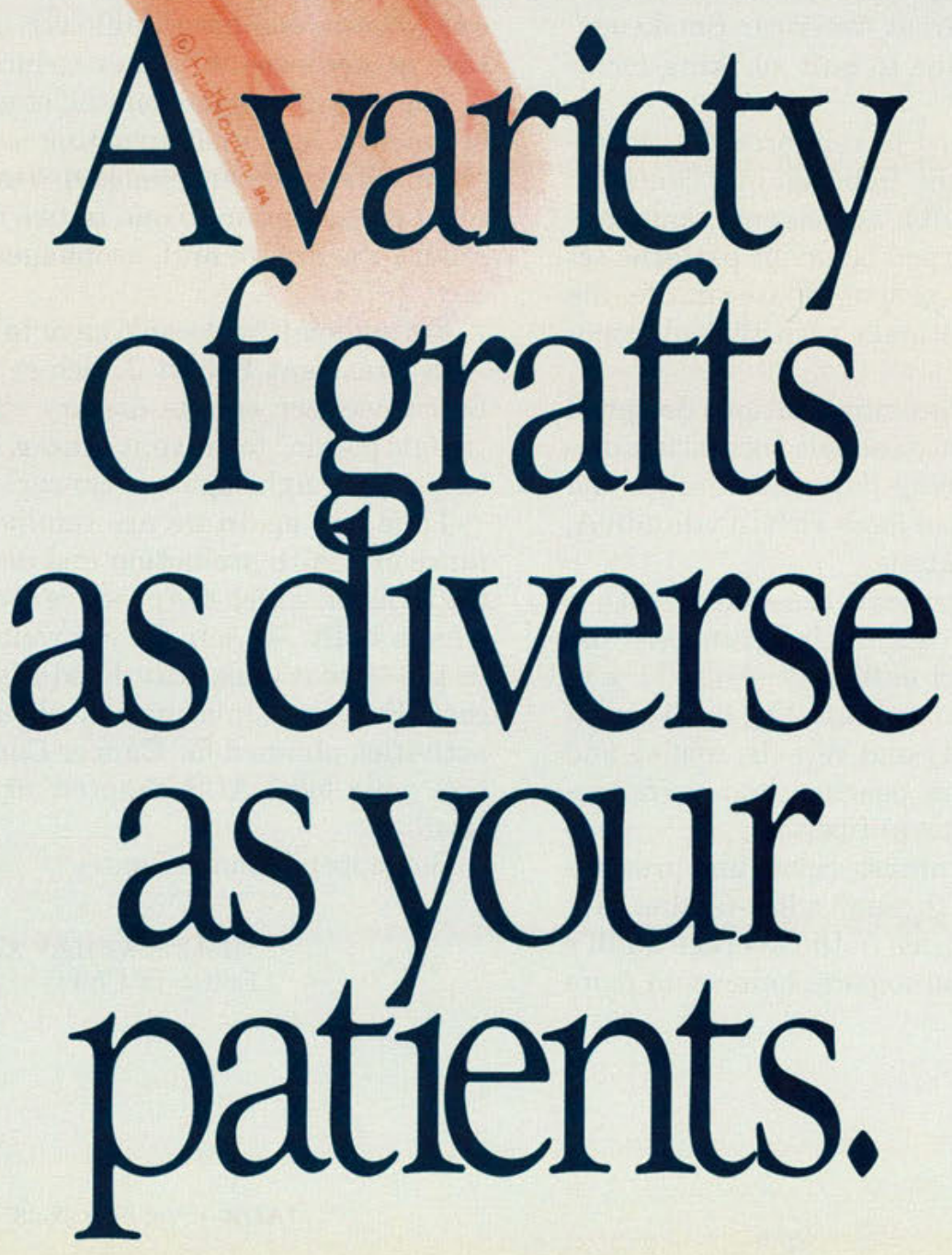




\section{Tapered.}

Hinsdale and colleagues report that, when using this

graft, they have seen a reduction in thrombosis

and arterial steal in the elderly population. ${ }^{3}$ Bell and Rosental write

"...the $4 \mathrm{~mm}$ taper has virtually eliminated high-flow situations..."

The tapered graft for angioaccess is available in $4-6 \mathrm{~mm}$, $4-7 \mathrm{~mm}$ and $4-7 \mathrm{~mm}$ diameters with removable rings.

3. Hinsdale JG, Lipkowitz GS, Hoover EL.

Vascular access for hemodialysis in the elderly:

Results and perspectives in a geriatric population.

Dial Transpl 1985; 14:560-565.

4. Bell DD and Rosental JJ. Arteriovenous graft life in chronic hemodialysis: A need for prolongation. Arch Surg 1988; 123:1169-1172.

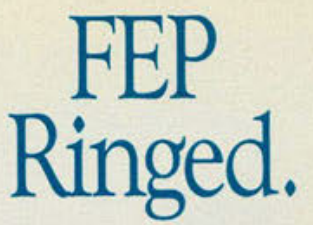

Many surgeons feel that the $5 \mathrm{~cm}$ of rings at the center of the graft help prevent any possible kink at the apex of a looped graft. The offset $7 \mathrm{~cm}$ of rings are ideal when crossing the elbow. These configurations are available in $6 \mathrm{~mm}$ diameter.

For over 14 years, GORE-TEX ${ }^{\circledR}$

Vascular Grafts have been used successfully

for angioaccess.

Over one million implants have proven that the grafts provide the durability necessary for a variety of patient needs.
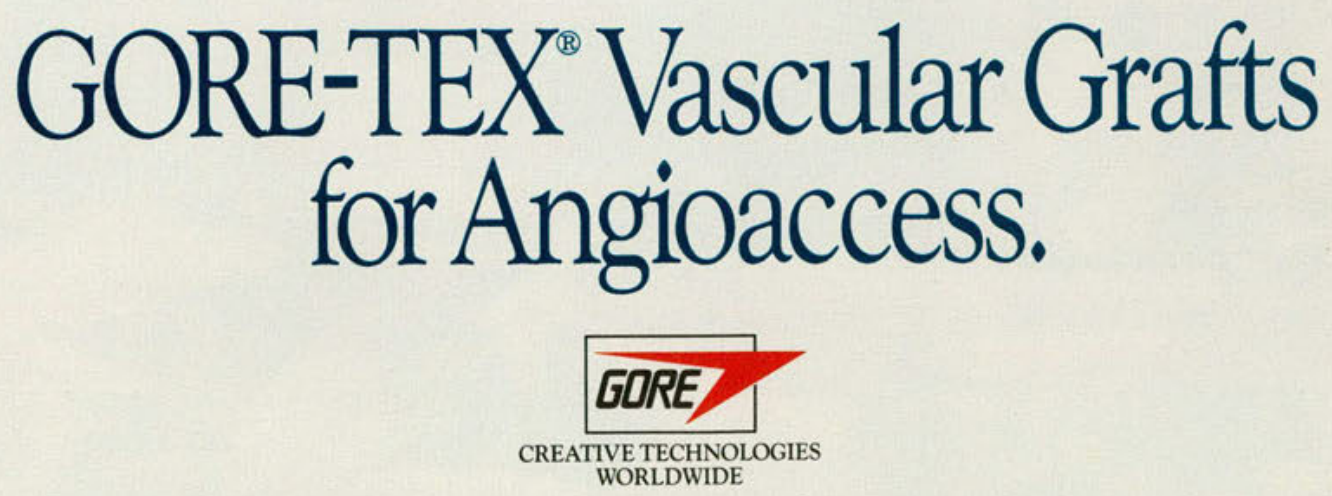

W.L. Gore \& Associates, Inc.

Post Office Box 200,1500 North 4th Street

Flagstaff, AZ 86002

For ordering or further information call: 800-528-8763

Ask for Department 31-F 


\section{HIS HISTORY \\ OF ULCER ONCE MEANT A FUTURE WITHOUT NSAIDS :}

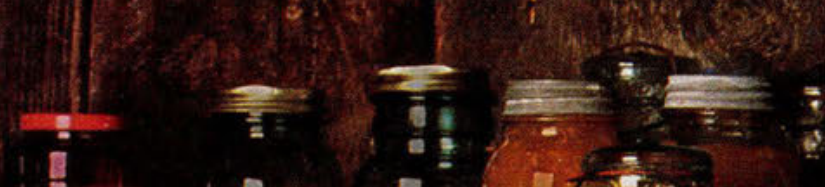
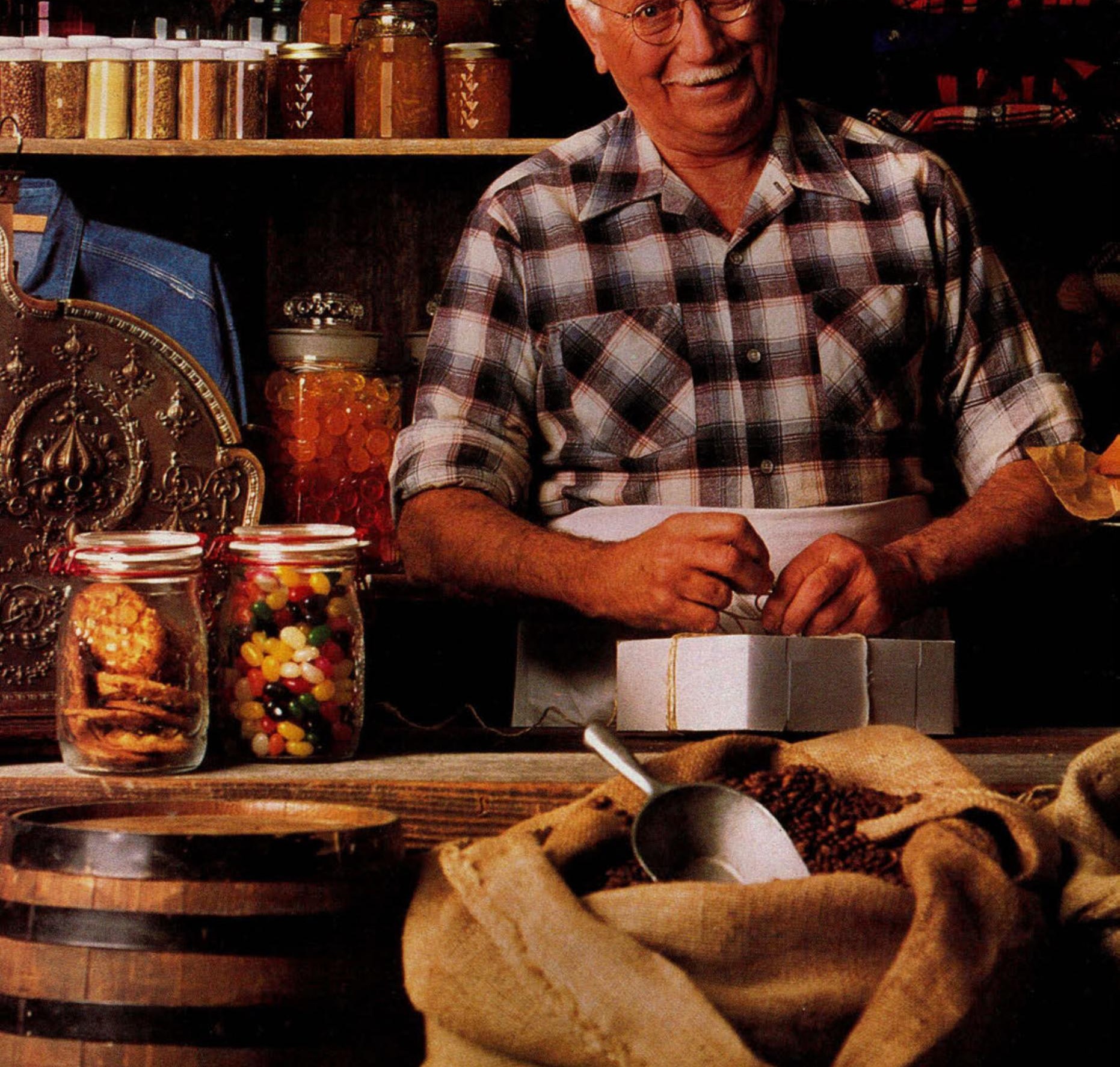


\section{TODAY, CYTOTEC REDUCES THE RISK OF GASTRIC ULCER FOR THE HIGH-RISK PATIENT}

Discontinuing therapy often seemed the only alternative for patients who developed gastric ulcers from chronic NSAID therapy.

Cytotec is indicated for those patients At high risk of experiencing gastric ulceration, e.g., those with a history of ulcer.

- At high risk of gastric ulcer complications, e.g., the elderly or those with concomitant debilitating disease.

\section{For NSAID users at high risk, prescribe}

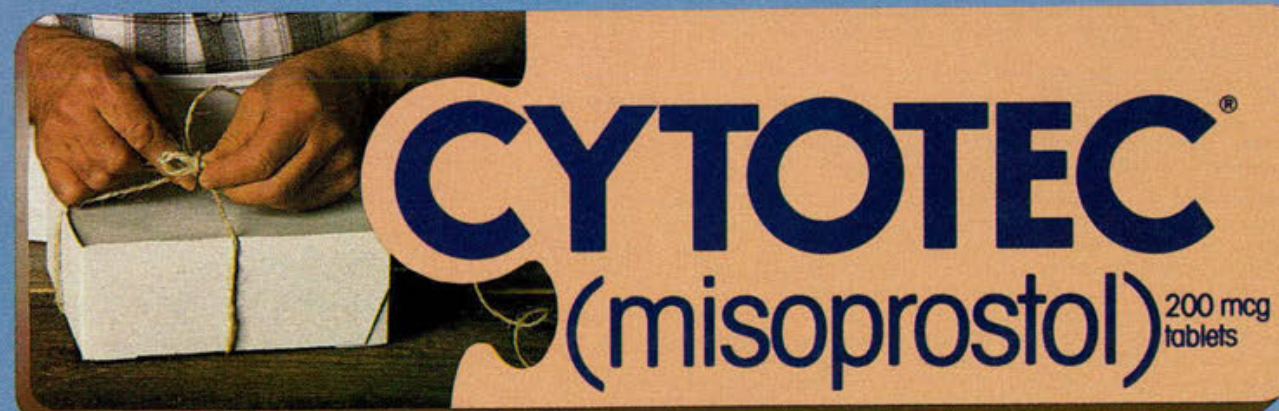

Please see adjoining page for brief summary of prescribing information. 


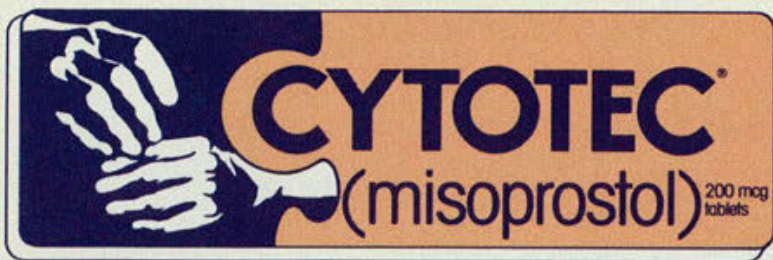

Considerations before you prescribe Cytotec

- Because of its abortifacient property, Cytotec should not be prescribed for women who are pregnant. Patients must be advised of the abortifacient property and warned not to give the drug to others.
Cytotec should be used in a woman of childbearing potential only if she is using effective contraceptive measures, has received oral and written warnings concerning the hazards of misoprostol, has had a negative serum pregnancy test within two weeks prior to beginning therapy, and will begin therapy only on the second or third day of the next menstrual period.

- Some patients may experience transient diarrhea, which usually resolves in about a week, or abdominal discomfort. Abdominal discomfort may persist in the absence of gastric ulceration

\section{BRIEF SUMMARY}

CONTRAINDICATIONS AND WARNINGS: Cytotec (misoprostol) is contraindicated, because of its abortifacient property, in women who are pregnant. (See Precautions.) Patients must be advised of the abortifacient property and warned not to give the drug to others. Cytotec should not be used in women of childbearing potential unless the patient requires nonsteroidal anti-inflammatory drug (NSAID) therapy and is at high risk of complications from gastric ulcers associated with use of the NSAID, or is at high risk of developing gastric ulceration. In such patients, Cytotec may be prescribed if the patient

- is capable of complying with effective contraceptive measures

- has received both oral and written warnings of the hazards of misoprostol the risk of possible contraception failure, and the danger to other women of childbearing potential should the drug be taken by mistake.

- has had a negative serum pregnancy test within two weeks prior to beginning therapy.

- will begin Cytotec only on the second or third day of the next normal menstrual period.

INDICATIONS AND USAGE: Cytotec (misoprostol) is indicated for the prevention of NSAID (nonsteroidal anti-inflammatory drugs, including aspirin)-induced gastric ulcers in patients at high risk of complications from gastric ulcer, eg, the elderly and patients with concomitant debilitating disease, as well as patients at high risk of developing gastric ulceration, such as patients with a history of ulcer. Cytotec has not been shown to prevent duodenal ulcers in patients taking NSAIDs. Cytotec should be taken for the duration of NSAID therapy. Cytotec has been shown to prevent gastric ulcers in controlled studies of three months' duration. It had no effect, compared to placebo, on gastrointestinal pain or discomfort associated with NSAID use.

CONTRAINDICATIONS: SEe boXed CONTRAINDICATIONS AND WARNINGS

Cytotec should not be taken by anyone with a history of allergy to prostaglandins. WARNINGS: See boxed CONTRAINDICATIONS AND WARNINGS

PRECAUTIONS

Information for patients: Cytotec is contraindicated in women who are pregnant and should not be used in women of childbearing potential unless the patient requires nonsteroidal anti-inflammatory drug (NSAID) therapy and is at high risk of complications from gastric ulcers associated with the use of the NSAID, or is at high risk of developing gastric ulceration. Women of childbearing potential should be told that they must not be pregnant when Cytotec therapy is initiated, and that they must use an effective contraception method while taking Cytotec.

an effective contraception method while taking Cytotec.
See boxed CONTRAINDICATIONS AND WARNINGS.

Patients should be advised of the following

Cytotec is intended for administration along with nonsteroidal anti-inflammatory drugs (NSAIDs), including aspirin, to decrease the chance of developing an NSAIDinduced gastric ulcer.

Cytotec should be taken only according to the directions given by a physician

If the patient has questions about or problems with Cytotec, the physician should be contacted promptly.

THE PATIENT SHOULD NOT GIVE CYTOTEC TO ANYONE ELSE. Cytotec has been prescribed for the patient's specific condition, may not be the correct treatment for another person, and may be dangerous to the other person if she were to become pregnant.

The Cytotec package the patient receives from the pharmacist will include a leaflet containing patient information. The patient should read the leaflet before taking Cytotec and each time the prescription is renewed because the leaflet may have been revised.

Keep Cytotec out of the reach of children

SPECIAL NOTE FOR WOMEN: Cytotec must not be used by pregnant women. Cytotec may cause miscarriage. Miscarriages caused by Cytotec may be incomplete, which could lead to potentially dangerous bleeding. hospitalization, surgery, infertility, or maternal or fetal death.

Drug interactions: See Clinical Pharmacology. Cytotec has not been shown to interfere with the beneficial effects of aspirin on signs and symptoms of rheumatoid arthritis. Cytotec does not exert clinically significant effects on the absorption, blood levels, and antiplatelet effects of therapeutic doses of aspirin. Cytotec has no clinically significant effect on the kinetics of diclofenac or ibuprofen.

Animal toxicology: A reversible increase in the number of normal surface gastric epithelial cells occurred in the dog, rat, and mouse. No such increase has been observed in humans administered Cytotec for up to one year.

An apparent response of the female mouse to Cytotec in long-term studies at 100 to 1000 times the human dose was hyperostosis, mainly of the medulla of sternebrae Hyperostosis did not occur in long-term studies in the dog and rat and has not been seen in humans treated with Cytotec.

Carcinogenesis, mutagenesis, impairment of fertility: There was no evidence of an effect of Cytotec on tumor occurrence or incidence in rats receiving daily doses up to 150 times the human dose for 24 months. Similarly, there was no effect of Cytotec on tumor occurrence or incidence in mice receiving daily doses up to 1000 times the human dose for 21 months. The mutagenic potential of Cytotec was tested in several in vitro assays, all of which were negative.

Misoprostol, when administered to breeding male and female rats at doses 6.25 times to 625 times the maximum recommended human therapeutic dose, produced dose-related pre- and post-implantation losses and a significant decrease in the number of live pups born at the highest dose. These findings suggest the possibility of a general adverse effect on fertility in males and females.

Pregnancy: Pregnancy Category $\mathrm{x}$. See boxed CONTRAINDICATIONS AND

Nonteratogenic effects: Cytotec may endanger pregnancy (may cause miscarriage) and thereby cause harm to the fetus when administered to a pregnant woman. Cytotec produces uterine contractions, uterine bleeding, and expulsion of the products of conception. Miscarriages caused by Cytotec may be incomplete. In studies in women undergoing elective termination of pregnancy during the first trimester. Cytotec caused partial or complete expulsion of the products of conception in $11 \%$ of the subjects and increased uterine bleeding in $41 \%$. If a woman is or becomes pregnant while taking this drug, the drug should be discontinued and the patient apprised of
whent while taking this drug, the drug
the potential hazard to the fetus.

the potential hazard to the fetus. doses 625 and 63 times the human dose, respectively.

Nursing mothers: See Contraindications. Cytotec should not be administered to nursing mothers because the potential excretion of misoprostol acid could cause significant diarrhea in nursing infants.

Pediatric use: Safety and effectiveness in children below the age of 18 years have not been established.

ADVERSE REACTIONS: The following have been reported as adverse events in subjects receiving Cytotec:

Gastrointestinal: The most frequent gastrointestinal adverse events were diarrhea and abdominal pain. The incidence of diarrhea ranged up to $40 \%$ but averaged $13 \%$ in clinical trials.

Diarrhea was dose related and usually developed early in the course of therapy (after 13 days), usually was self-limiting (often resolving after 8 days), but sometimes required discontinuation of Cytotec (2\% of the patients). Rare instances of profound diarrhea leading to severe dehydration have been reported. Patients with an underlying condition such as inflammatory bowel disease, or those in whom dehydration, were it to occur, would be dangerous, should be monitored carefully if Cytotec is prescribed. The incidence of diarrhea can be minimized by administering after meals and at bedtime, and by avoiding coadministration of Cytotec with magnesium-containing antacids

Gynecological: Women who received Cytotec during clinical trials reported the following gynecological disorders: spotting $(0.7 \%)$, cramps $(0.6 \%)$, hypermenorrhea $(0.5 \%)$, menstrual disorder $(0.3 \%)$ and dysmenorrhea $(0.1 \%)$. Postmenopausal vaginal bleeding may be related to Cytotec administration. If it occurs, diagnostic workup should be undertaken to rule out gynecological pathology.

Elderly: There were no significant differences in the safety profile of Cytotec in approximately 500 ulcer patients who were 65 years of age or older compared with younger patients.

Additional adverse events which were reported are categorized as follows:

Incidence greater than $1 \%$ : In clinical trials, the following adverse reactions were reported by more than $1 \%$ of the subjects receiving Cytotec and may be causally related to the drug: nausea $(3.2 \%)$, flatulence $(2.9 \%)$, headache $(2.4 \%)$, dyspepsia $(2.0 \%)$, vomiting $(1.3 \%)$, and constipation $(1.1 \%)$. However, there were no significant differences between the incidences of these events for Cytotec and placebo.

Causal relationship unknown: The following adverse events were infrequently reported. Causal relationships between Cytotec and these events have not been established but cannot be excluded: aches/pains, asthenia, fatigue, fever, rigors, weight changes, rash, dermatitis, alopecia, pallor, breast pain, abnormal taste, abnormal vision, conjunctivitis, deafness, tinnitus, earache, upper respiratory tract infection, bronchitis, bronchospasm, dyspnea, pneumonia, epistaxis, chest pain edema, diaphoresis, hypotension, hypertension, arrhythmia, phlebitis, increased cardiac enzymes, syncope, GI bleeding. GI inflammation/infection, rectal disorder, abnormal hepatobiliary function, gingivitis, reflux, dysphagia, amylase increase, glycosuria, gout, increased nitrogen, increased alkaline phosphatase, polyuria, dysuria, uria, gout, increased nitrogen, increased alkaline phosphatase, polyuria, dysuria,
hematuria, urinary tract infection, anxiety, change in appetite, depression, drowsiness. dizziness, thirst, impotence, loss of libido, sweating increase, neuropathy, neurosis arthralgia, myalgia, muscle cramps, stiffness, back pain, anemia, abnormal differential thrombocytopenia, purpura, ESR increased.

Important note: Complete prescribing information should be consulted prior to use.

DOSAGE AND ADMINISTRATION: The recommended adult oral dose of Cytotec for the prevention of NSAID-induced gastric ulcers is $200 \mathrm{mcg}$ four times daily with food. If this dose cannot be tolerated, a dose of $100 \mathrm{mcg}$ can be used. Cytotec should be taken for the duration of NSAID therapy as prescribed by the physician. Cytotec should be taken with a meal, and the last dose of the day should be at bedtime

Renal impairment: Adjustment of the dosing schedule in renally impaired patients is not routinely needed, but dosage can be reduced if the 200 -mcg dose is not tolerated.

Address medical inquiries to

$1 / 23 / 90 \cdot P 90-R 114 V$

Medical \& Scientific Information Department

4901 Searle Parkway

Skokie, IL 60077 